\title{
Light-by-light scattering and spacetime noncommutativity
}

\author{
Raul Horvat@, ${ }^{1, *}$ Duško Latas $\odot,{ }^{2, \dagger}$ Josip Trampetić $\odot,{ }^{1,3, *}$ and Jiangyang You $\odot^{4, \S}$ \\ ${ }^{1}$ Ruđer Bošković Institute, Bijenička 54, 10000 Zagreb, Croatia \\ ${ }^{2}$ University of Belgrade, Faculty of Physics, P.O Box 44, 11000 Belgrade, Serbia \\ ${ }^{3}$ Max-Planck-Institut für Physik, (Werner-Heisenberg-Institut), Föhringer Ring 6, \\ D-80805 München, Germany \\ ${ }^{4}$ Ruđer Bošković Institute, Division of Physical Chemistry, Bijenička 54, \\ 10000 Zagreb, Croatia
}

(Received 14 February 2020; accepted 7 May 2020; published 27 May 2020)

\begin{abstract}
The aim of this article is to explore a potential usability of a photon-photon self-interaction from the noncommutative quantum electrodynamics (NCQED) in the case of light-by-light scattering $(\gamma \gamma \rightarrow \gamma \gamma)$ in ultraperipheral $\mathrm{Pb}+\mathrm{Pb}$ collisions, a reaction measured recently by ATLAS and planned for future experiments in hadron-hadron colliders. We compute the total cross section from both, the full one-loop standard model (SM) and the tree-level NCQED amplitudes, in the equivalent photon approximation with impact parameters, for various noncommutative scales, $\Lambda_{\mathrm{NC}}$, and incoming nuclear spin-energy combinations. We find that NCQED contribution to the cross section has considerable increase at diphoton invariant mass range higher than $\Lambda_{\mathrm{NC}}$, while the SM contribution is strongly suppressed in such region. Our results show that the current ATLAS $\sqrt{\mathrm{s}_{\mathrm{NN}}}=5.02 \mathrm{TeV}$ experiment can only probe $\Lambda_{\mathrm{NC}}<$ $100 \mathrm{GeV}$ region. On the other hand, future hadron-hadron collider proposals could have the potential to extend to $\Lambda_{\mathrm{NC}} \lesssim 300 \mathrm{GeV}$ region, making the performance of $\mathrm{PbPb} \rightarrow \mathrm{PbPb} \gamma \gamma$ scattering on testing spacetime noncommutativity close to that of the previously proposed photon-photon mode of linear electronpositron collider.
\end{abstract}

DOI: 10.1103/PhysRevD.101.095035

Space-time noncommutativity and quantizations of the electromagnetic field in such noncommutative (NC) space, was an original idea to cure UV divergences plaguing quantum field theory, suggested by Heisenberg to Peierls, followed to Pauli, and finally executed a bit later by Oppenheimer's graduate student, Snyder [1,2]. Nowadays such models appear quite naturally in certain limits of string theory in the presence of a background $B^{\mu \nu}$ field. Specifically, a low-energy limit is identified where the entire boson-string dynamics in a Neveu-Schwartz condensate is described by a minimally coupled supersymmetric gauge theory on noncommutative space [3] such that the mathematical framework of NC geometry/field theory $[4,5]$ does apply.

Of various types of noncommutative space-time structures the one generated by a constant real antisymmetric

\footnotetext{
raul.horvat@irb.hr

†latas@ipb.ac.rs

trampeti@mppmu.mpg.de

§iangyang.you@irb.hr
}

Published by the American Physical Society under the terms of the Creative Commons Attribution 4.0 International license. Further distribution of this work must maintain attribution to the author(s) and the published article's title, journal citation, and DOI. Funded by SCOAP ${ }^{3}$. matrix $\theta^{\mu \nu}$ of dimension length ${ }^{2}$, i.e. the Moyal space, has attracted a large amount of research interests for several crucial reasons: It emerges from string theory in a constant $B^{\mu \nu}$ field background [3]. Pertubatively quantized gauge theories can be constructed through the nonlocal MoyalWeyl star $(\star)$-product realization [6], and the existence of intriguing mathematical structure called Seiberg-Witten (SW) map [3]. Thanking to these properties, NCQFT and related topics have been constantly investigated throughout time for over two decades now [6-31]. Particle physics models on NC space-time close to the existing standard model have been successfully in via NC gauge theories in Moyal space [8-10,21,31]. Various bounds on $\mathrm{NC}$ scales have been established over time through these models [7,11,13,17,18,20,22,23,31].

One direct consequence of noncommutativity is that gauge bosons (i.e., photons) in a $\mathrm{NC} \mathrm{U}(1)$ gauge theory become self-coupled. This property has very influential consequences in the $\theta$-exact NCQFT which are still under investigation now [6,12,14-16,26-29]. NC photon selfcoupling also induces nontrivial tree-level corrections to photon related processes like pair production, pair annihilation, Compton scattering, and most importantly, the tree-level photon-photon light-by-light (L-by-L) scattering process. The latter is considered a good candidate process in search for possible signal of space-time 
noncommutativity because the same process is suppressed at tree-level in standard model due to the Landau-Young theorem.

Studies on light-by-light scattering used to focus on the photon-photon mode of linear electron-positron collider [31-35]. Recently a new method for observing light-bylight scattering from the ultraperipheral heavy ion scattering has attracted much attention from society [36-44]. In such scenario the electromagnetic field of highly relativistic incoming heavy ions $\mathrm{X}\left(\mathrm{X}={ }^{208} \mathrm{~Pb}\right.$, $\left.{ }^{197} \mathrm{Au}, \ldots ..\right)$ is approximated as distribution of almost onshell photons (equivalent photon approximation). In short, the process $\mathrm{XX} \rightarrow \mathrm{XX} \gamma \gamma$ can then be treated as convolution of incoming photon distributions and $\gamma \gamma \rightarrow \gamma \gamma$ scattering [36-41,43,44]. Initial observation of such processes on the ATLAS experiment of LHC strongly boosted the interest on light-by-light scattering $[39,40]$. Heavy ion collisions are also considered in the proposals for next generation circular hadron collider(s) [45-47].

Using peripheral scattering scenario as a test bed for various beyond standard model theories have also been frequently considered. The theories engaged in testing the photon self-coupling in ultraperipheral $\mathrm{PbPb} \rightarrow \mathrm{PbPb} \gamma \gamma$ collisions include perturbatively expanded Born-Infeld theory, some nonlinear corrections to Maxwell electrodynamics, and local Lorentz violating operators [41-43], but not the nonlocal Lorentz violating operators in NCQED. In this work, we present a new computation for the tree level photon-photon scattering in $\mathrm{LHC} \mathrm{Pb}-\mathrm{Pb}$ collisions in the minimal $\theta$-exact SW mapped NCQED model $[12,14]$, in scenarios associated to this and next generation hadron collider(s) and estimate the capability of these scenarios in probing the NC scales.

Under the assumption that main deviations from the SM in the exclusive $\gamma \gamma \rightarrow \gamma \gamma$ scattering measurements originated from the NCQED, in this paper we continue to search for the scale $\Lambda_{\mathrm{NC}}$ of the NC spacetime by using our $\theta$-exact minimal gauge sector action

$$
\begin{aligned}
S_{\text {gauge }}^{\min } & =\int-\frac{1}{2} F^{\mu \nu} \star F_{\mu \nu}, \\
F_{\mu \nu} & =\partial_{\mu} A_{\nu}-\partial_{\nu} A_{\mu}-i\left(A_{\mu} \star A_{\nu}-A_{\nu} \star A_{\mu}\right),
\end{aligned}
$$

given in terms of the NC gauge field $A_{\mu}$. The connection between the noncommutative and commutative fields is given by the $\theta$-exact Seiberg-Witten maps. Perturbative quantization is done by the standard Becchi-Rouet-StoraTyutin procedure [26]. Note that our approach here does not involve an expansion over the deformation parameter $\theta$ itself. Therefore our results below do not have an upper energy limit by the noncommutative scale.

Through lengthy and straightforward diagrammatic computation one can prove that the NC scattering amplitudes are invariant under the $\theta$-exact SW maps, i.e., all additional contributions induced in the action (1) by the SW map cancel out [48].

The $\gamma \gamma \rightarrow \gamma \gamma$ scattering amplitudes in NCQED without SW map can be solved from a color- $-\star$-product equivalence between the ordinary $\mathrm{U}(\mathrm{N})$ and the Moyal NC U(1) gauge theories [48-50],

$$
\operatorname{tr} \prod_{i=1}^{n} T^{\alpha_{i}} \Leftrightarrow \exp \left(-\frac{i}{2} \sum_{i=1}^{n} k_{2 i-1} \theta k_{2 i}\right)
$$

By this means the noncommutative structure decouples with the $\star$-product ordered amplitudes, making the latter identical to the corresponding QCD color-ordered amplitudes. After a summation over contributing color orders, we reach the L-by-L helicity scattering amplitudes of NCQED [31]. Of all possible helicity combinations only three are nonzero and independent $[31,48]$ :

$$
\begin{aligned}
& M_{\mathrm{NC}}^{++++}=32 \pi \alpha\left(\frac{s}{u} \sin \frac{k_{1} \theta k_{2}}{2} \sin \frac{k_{3} \theta k_{4}}{2}-\frac{s^{2}}{t u} \sin \frac{k_{1} \theta k_{4}}{2} \sin \frac{k_{2} \theta k_{3}}{2}\right), \\
& M_{\mathrm{NC}}^{+-+-}=32 \pi \alpha\left(\frac{t^{2}}{s u} \sin \frac{k_{1} \theta k_{2}}{2} \sin \frac{k_{3} \theta k_{4}}{2}-\frac{t}{u} \sin \frac{k_{1} \theta k_{4}}{2} \sin \frac{k_{2} \theta k_{3}}{2}\right), \\
& M_{\mathrm{NC}}^{++--}=32 \pi \alpha\left(\frac{u}{s} \sin \frac{k_{1} \theta k_{2}}{2} \sin \frac{k_{3} \theta k_{4}}{2}-\frac{u}{t} \sin \frac{k_{1} \theta k_{4}}{2} \sin \frac{k_{2} \theta k_{3}}{2}\right) .
\end{aligned}
$$

We then evaluate the NCQED contribution to the ultraperipheral $\mathrm{PbPb} \rightarrow \mathrm{PbPb} \gamma \gamma$ process in the equivalent photon approximation $[37,44]$. To do this we sum over the tree-level NCQED and one-loop standard model amplitudes [33-35] to evaluate the exclusive $\gamma \gamma \rightarrow \gamma \gamma$ differential cross in polar coordinates:

$$
\frac{d \sigma\left(\omega_{1}, \omega_{2}, \vartheta, \varphi\right)}{d \Omega}=\frac{1}{(16 \pi)^{2}} \frac{1}{\left(\omega_{1}(1-\cos \vartheta)+\omega_{2}(1+\cos \vartheta)\right)^{2}} \sum_{h}\left|M_{\mathrm{SM}}^{h}+M_{\mathrm{NC}}^{h}\right|^{2}, \quad d \Omega=\sin \vartheta d \vartheta d \varphi .
$$


Next we compute the total $\mathrm{PbPb} \rightarrow \mathrm{PbPb} \gamma \gamma$ cross section by convoluting the exclusive differential cross section with the photon distribution of the equivalent photon beams, i.e., the photon number function $N\left(\omega_{i},\left|\vec{b}_{i}\right|\right)(i=1,2)$. Here 2D vectors $\vec{b}_{i}$ are the impact parameters $[37,44]$ that mark the positions of the ions from the position of impact in the plane perpendicular to the beam direction. Peripheral nature of the collision requires $\left|\vec{b}_{1}-\vec{b}_{2}\right|>R_{\min }=2 R_{\mathrm{N}}$ to exclude direct collision between nuclei when integrating over beams. The incoming photon spectrum space $\left(\omega_{1}, \omega_{2}\right)$ is usually reparametrized by the diphoton invariant mass $m_{\gamma \gamma}=\sqrt{4 \omega_{1} \omega_{2}}$ and rapidity $Y=\frac{1}{2} \ln \frac{\omega_{1}}{\omega_{2}}$. Taking all these into account the cross section of $\mathrm{PbPb} \rightarrow \mathrm{PbPb} \gamma \gamma$ is expressed in the fiducial phase space as an eightfold integral:

$$
\sigma=\frac{1}{2} \int d m_{\gamma \gamma} d Y \frac{d^{2} L_{\gamma \gamma}}{d m_{\gamma \gamma} d Y} d \Omega \frac{d \sigma\left(\omega_{1}, \omega_{2}, \vartheta, \varphi\right)}{d \Omega},
$$

by convoluting (4) with luminosity function $\frac{d^{2} L_{\gamma \gamma}}{d m_{\gamma \gamma} d Y}$ :

$$
\frac{d^{2} L_{\gamma \gamma}}{d m_{\gamma \gamma} d Y}=\frac{4 \pi}{m_{\gamma \gamma}} \int_{0}^{\infty} d\left|\vec{b}_{1}\right| \int_{0}^{\infty} d\left|\vec{b}_{2}\right| \int_{0}^{2 \pi} d \phi N\left(\omega_{1},\left|\vec{b}_{1}\right|\right) N\left(\omega_{2},\left|\vec{b}_{2}\right|\right) \Theta\left(\sqrt{\left|\vec{b}_{1}\right|^{2}+\left|\vec{b}_{2}\right|^{2}-2\left|\vec{b}_{1}\right|\left|\vec{b}_{2}\right| \cos \phi}-R_{\min }\right),
$$

where we take $R_{\min }=2 R_{\mathrm{Pb}}=14 \mathrm{fm} \simeq 71 \mathrm{GeV}^{-1}$. In this work the fourfold integration over impact parameters $\vec{b}_{1}$ and $\vec{b}_{2}$ is performed with the photon number function from monopole form factor $[37,44]$

$$
N(\omega, b)=\frac{Z^{2} \alpha}{\pi^{2}}\left(\frac{\omega}{\gamma} K_{1}\left(\frac{\omega b}{\gamma}\right)-\sqrt{\frac{\omega^{2}}{\gamma^{2}}+\xi^{2}} \cdot K_{1}\left(b \sqrt{\frac{\omega^{2}}{\gamma^{2}}+\xi^{2}}\right)\right)^{2}, \quad \gamma=\frac{\sqrt{s_{\mathrm{NN}}}}{2 m_{u}} .
$$

In the above $K_{1}$ is the modified Bessel function of 2 nd kind, $\xi=0.088 \mathrm{GeV}$ for ${ }^{208} \mathrm{~Pb}$ ion, $\gamma$ is the ion Lorenz factor and $m_{u}=0.931 \mathrm{GeV}$ is the atomic mass unit.

Remaining integration over $d m_{\gamma \gamma} d Y d \Omega$ has to be performed over an domain defined according to the experimental selection rules. For a typical cut which requires transverse energy $E_{T} \geq E_{0}$ and both outgoing particles to bear absolute pseudorapidities $\left|y_{3,4}\right| \leq y_{0}$, the full integral domain is defined as follows:

$$
\begin{aligned}
\varphi & \in[0,2 \pi], \quad|\cos \vartheta| \leq \frac{e^{2 y_{0}}-1}{e^{2 y_{0}}+1}, \quad m_{\gamma \gamma} \geq 2 E_{0}, \\
Y & \in\left[\frac{1}{2}\left(\frac{1}{2} \ln \frac{1+\cos \vartheta}{1-\cos \vartheta}-y_{0}\right), \frac{1}{2}\left(y_{0}-\frac{1}{2} \ln \frac{1+\cos \vartheta}{1-\cos \vartheta}\right)\right] \\
& \cap\left[\frac{1}{2} \ln \frac{1+\cos \vartheta}{1-\cos \vartheta}+\ln \left(\frac{m_{\gamma \gamma}}{2 E_{0}}-\sqrt{\frac{m_{\gamma \gamma}^{2}}{4 E_{0}^{2}}-1}\right), \frac{1}{2} \ln \frac{1+\cos \vartheta}{1-\cos \vartheta}+\ln \left(\frac{m_{\gamma \gamma}}{2 E_{0}}+\sqrt{\frac{m_{\gamma \gamma}^{2}}{4 E_{0}^{2}}}-1\right)\right] .
\end{aligned}
$$

From $[39,40]$ we have $E_{0}=3 \mathrm{GeV}$ and $y_{0}=2.37$ to define the ATLAS cuts. These cuts and $\gamma=2693$ for ATLAS experiment with lead ion center of mass energy $\sqrt{s_{\mathrm{NN}}}=$ $5.02 \mathrm{TeV}$ [40] allows us to estimate full SM one-loop contributions to the fiducial cross section: $\sigma_{\mathrm{SM}}(\mathrm{PbPb} \rightarrow$ $\mathrm{PbPb} \gamma \gamma)=57 \mathrm{nb}$, comparable to the previously reported values of $\simeq 50 \mathrm{nb}$ [40].

We then numerically compute combined contributions from the SM one-loop and the NCQED tree-level $\gamma \gamma \rightarrow \gamma \gamma$ amplitudes to the $\mathrm{PbPb} \rightarrow \mathrm{PbPb} \gamma \gamma$ collision at several $\Lambda_{\mathrm{NC}}$ values. The NC tensor $\theta^{\mu \nu}$ is set to have only space-space components $\theta^{i 3} \neq 0$ for simplicity. The third axis is set to be parallel to the beam, as usual. The result indicates that NC contribution takes place mainly at diphoton mass range considerably higher than the dominating part of the SM contribution (Fig. 1) when $\Lambda_{\mathrm{NC}}$ is so valued that NC contribution to the cross section is comparable with that of SM (Table I). We therefore consider that the high diphoton

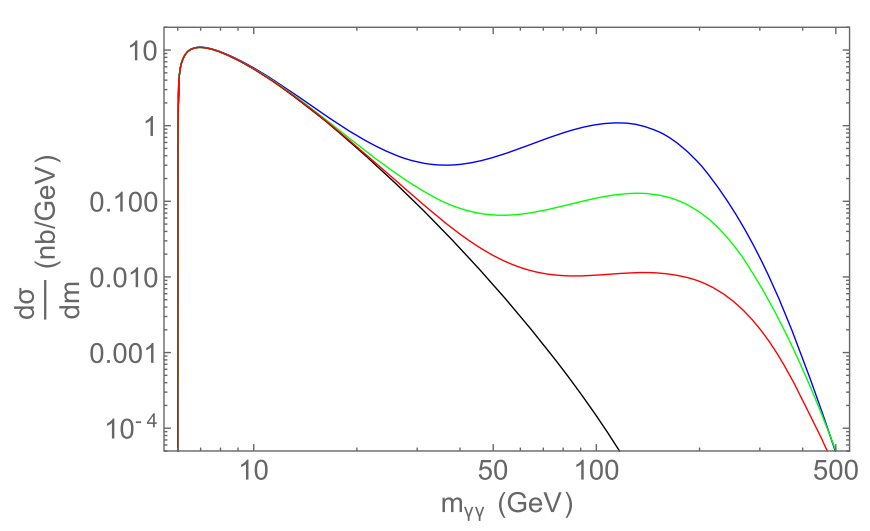

FIG. 1. Cross section versus diphoton invariant mass distribution in the $\mathrm{PbPb} \rightarrow \mathrm{PbPb} \gamma \gamma$ experiment with the ATLAS cuts at $\sqrt{s_{\mathrm{NN}}}=5.02 \mathrm{TeV}$, for the pure $\mathrm{SM}$ (black) as well as the $\mathrm{SM}+\mathrm{NCQED}$ with $\Lambda_{\mathrm{NC}}$ values 53 (blue), 72 (green) and 100 (red) $\mathrm{GeV}$. 
TABLE I. Summary of the predicted $\sigma_{\mathrm{SM}}$ and $\sigma_{\mathrm{NCQED}}=\sigma_{\mathrm{NC}}+$ $\sigma_{\mathrm{NC}^{2}}$ contributions to $\mathrm{PbPb} \rightarrow \mathrm{PbPb} \gamma \gamma$ fiducial cross sections for the ATLAS cuts at $\sqrt{s_{\mathrm{NN}}}=5.02 \mathrm{TeV}$ and various $\Lambda_{\mathrm{NC}}$ values. $\left.\mathrm{m}_{\gamma \gamma}\right|_{\max }$ denotes the diphoton mass position of $\left.\frac{\mathrm{d} \sigma_{\mathrm{NCQED}}}{\mathrm{dm} \mathrm{m}_{\gamma \gamma}}\right|_{\max }$.

\begin{tabular}{|c|c|c|c|c|c|c|}
\hline $\begin{array}{l}\Lambda_{\mathrm{NC}} \\
(\mathrm{GeV})\end{array}$ & $\begin{array}{l}\sigma_{\mathrm{SM}} \\
(\mathrm{nb})\end{array}$ & $\begin{array}{l}\sigma_{\mathrm{NC}} \\
(\mathrm{nb})\end{array}$ & $\begin{array}{l}\sigma_{\mathrm{NC}{ }^{2}} \\
(\mathrm{nb})\end{array}$ & $\begin{array}{c}\sigma_{\mathrm{NCQED}} \\
(\mathrm{nb})\end{array}$ & $\left.\frac{\mathrm{d} \sigma_{\mathrm{NCQED}}}{\mathrm{dm}_{\gamma \gamma}}\right|_{\max }\left(\frac{\mathrm{nb}}{\mathrm{GeV}}\right)$ & $\begin{array}{c}\left.\mathrm{m}_{y \gamma}\right|_{\text {max }} \\
(\mathrm{GeV})\end{array}$ \\
\hline 53 & 57 & 12.1 & 125.5 & 137.2 & 1.09 & 115 \\
\hline 72 & 57 & 3.6 & 17.6 & 21.2 & 0.13 & 132 \\
\hline 100 & 57 & 1.0 & 1.8 & 2.8 & 0.011 & 138 \\
\hline
\end{tabular}

mass peak from the NCQED contribution determines the probability for the experiment to detect it. Our results presented in Table I and Fig. 1 show that diphoton invariant mass distribution peak values of $\sim(1,0.1,0.01) \frac{\mathrm{nb}}{\mathrm{GeV}}$ exist for $\Lambda_{\mathrm{NC}} \gtrsim(53,72,100) \mathrm{GeV}$, respectively. Given the current integrated luminosity accumulation speed $\left(\sim 1 \mathrm{nb}^{-1} / \mathrm{yr}\right)$ of current LHC and assuming that high luminosity (HL) LHC will reach about ten times of this value, we estimate that the current ATLAS experiment could only probe $\Lambda_{\mathrm{NC}}<100 \mathrm{GeV}$.

The existence of a second peak, with maxims at $\left.\mathrm{m}_{\gamma \gamma}\right|_{\max }$ reflects the evolution of noncommutative factors with respect to energy scales: When energy scales are much smaller than $\Lambda_{\mathrm{NC}}$, the NC factors are increasing as monomials with high power. Once the energy scales become larger than $\Lambda_{\mathrm{NC}}$ the $\mathrm{NC}$ factors become oscillatory and bounded. Consequently the NC amplitudes are very small at very low energies where SM contribution dominates, and then increase fast and compete with the exponentially decreasing luminosity factor [44] to give the rising side of the NC second peak. Once the energy scale goes beyond $\Lambda_{\mathrm{NC}}$ the NC amplitudes start to deviate from monomial increase, so that $\frac{\mathrm{d} \sigma}{\mathrm{dm}}$ falls down quickly. The subsequent oscillatory behaviors of the $\mathrm{NC}$ factors are fully suppressed

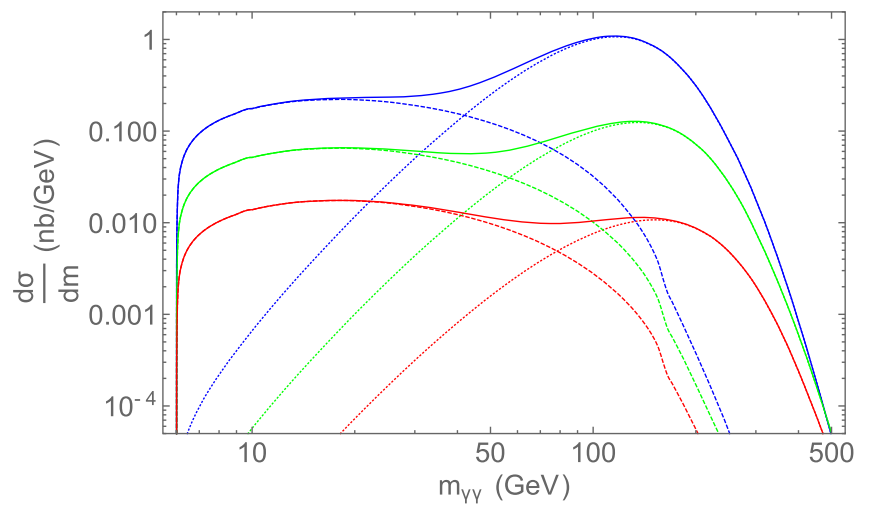

FIG. 2. NCQED related contributions to the cross section at $\sqrt{s_{\mathrm{NN}}}=5.02 \mathrm{TeV}$ versus diphoton invariant mass distributions under the ATLAS cuts conditions for $\Lambda_{\mathrm{NC}}$ values 53 (blue), 72 (green) and 100 (red) GeV. Dotted lines are pure noncommutative contribution $\left(\mathrm{NC}^{2}\right)$, dashed lines are $\mathrm{SM} \times \mathrm{NCQED}$ interference (NC), and solid lines are sum: $\mathrm{NC}+\mathrm{NC}^{2}=\mathrm{NCQED}$. by the luminosity function and cannot be seen in this scenario. Consequently, the $\mathrm{NC}^{2}$ contribution tends to reach a maximum at diphoton mass scales higher than corresponding NC contribution, as shown in Fig. 2. The latter also shows a kind of plateau in the plot $\frac{\mathrm{d} \sigma}{\mathrm{dm}_{\gamma \gamma}}$ because of the interference pattern. Matching the reported experimental total cross section of $78 \mathrm{nb}$ [40] by such combination yields a very small NC scale $\Lambda_{\mathrm{NC}}$ of about $72 \mathrm{GeV}$. This does not explain the experimental excess since the cross section induced by the NCQED amplitude mainly comes from the region $\mathrm{m}_{\gamma \gamma} \gtrsim 100 \mathrm{GeV}$, which turns out to be much above the experimentally measured excess of $\sim 30 \mathrm{GeV}$, as nicely presented by ATLAS Collaboration in Fig. 2(b) of [40].

Because of the limited probing capability of current ATLAS experiment, we move on to estimate potential improvements/enhancements one would expect from future generation circular collider(s). We consider proposals for next generation hadron collider Super Proton-Proton Collider (SppC) with energy $\sqrt{s_{\mathrm{pp}}}=70 \mathrm{TeV}$ [51], and from the Future Circular Collider (FCC) proposal up to $\sqrt{s_{\mathrm{pp}}}=100 \mathrm{TeV}$ [52-56], as well as a far future scenario with $\sqrt{s_{\mathrm{NN}}} \simeq 100 \mathrm{TeV}$. We assume that all the ATLAS cuts kinematics remains the same, except the energy scale/ Lorentz factor which scales up to 5, 7, or 20 times with respect to the current ATLAS value $\sqrt{s_{\mathrm{NN}}}=5.02 \mathrm{TeV}$, and estimate the NC scales $\Lambda_{\mathrm{NC}}$ corresponding to a high energy $\left.\frac{\mathrm{d} \sigma}{\mathrm{dm}_{y \gamma}}\right|_{\max }$ with $\sim 0.01 \mathrm{nb} / \mathrm{GeV}$ magnitude. Table II shows that $\Lambda_{\mathrm{NC}}$ are about 2.5, 3.1 and 5.2 times of the $\sim 100 \mathrm{GeV}$ limit for the ATLAS energy scale $5.02 \mathrm{TeV}$, respectively. Thus we conclude that future ultraperipheral heavy ion collision experiment(s) could probe $\Lambda_{\mathrm{NC}}$ scales close to L-by-L scattering in the photon-photon mode of a linear $\mathrm{e}^{-} \mathrm{e}^{+}$collider $(\sim 500 \mathrm{GeV})[31]$.

Our estimations show that like in the original ATLAS case (Figs. 1 and 2), NCQED contribution in the next generation hadron collider ultraperipheral heavy ion scattering scenarios also manifests as a second peak of $\frac{\mathrm{d} \sigma}{\mathrm{dm} \mathrm{m}_{r y}}$ at diphoton mass range moderately higher than $\Lambda_{\mathrm{NC}}$ (Fig. 3 ). In addition Fig. 3 transparently shows that the point at which NCQED starts decoupling from the SM, and the

TABLE II. Estimations for ATLAS $\mathrm{PbPb} \rightarrow \mathrm{PbPb} \gamma \gamma$ like experiments at higher energies. Here we made adjustments of the NC scale in a way to obtain $\left.\frac{\mathrm{d} \sigma_{\mathrm{NCQED}}}{\mathrm{dm}_{r \gamma}}\right|_{\max } \simeq 0.01\left(\frac{\mathrm{nb}}{\mathrm{GeV}}\right)$, at relevant $\left.\mathrm{m}_{\gamma \gamma}\right|_{\max }$ diphoton mass points given in this table.

\begin{tabular}{lrcrrrr}
\hline \hline $\begin{array}{l}\sqrt{\mathrm{s}_{\mathrm{NN}}} \\
(\mathrm{TeV})\end{array}$ & \multicolumn{1}{c}{$\gamma$} & $\begin{array}{c}\Lambda_{\mathrm{NC}} \\
(\mathrm{GeV})\end{array}$ & $\begin{array}{r}\sigma_{\mathrm{SM}} \\
(\mathrm{nb})\end{array}$ & $\begin{array}{c}\sigma_{\mathrm{NCQED}} \\
(\mathrm{nb})\end{array}$ & $\left.\frac{\mathrm{d} \sigma_{\mathrm{NCQED}}}{\mathrm{dm}_{\gamma \gamma}}\right|_{\max }\left(\frac{\mathrm{nb}}{\mathrm{GeV}}\right)$ & $\begin{array}{c}\left.\mathrm{m}_{\gamma \gamma}\right|_{\max } \\
(\mathrm{GeV})\end{array}$ \\
\hline 5.02 & 2693 & 100 & 57 & 2.8 & 0.011 & 138 \\
25.10 & 13465 & 257 & 178 & 6.6 & 0.011 & 567 \\
35.14 & 18851 & 311 & 211 & 7.9 & 0.010 & 737 \\
100.40 & 53860 & 523 & 336 & 16.9 & 0.011 & 1480 \\
\hline \hline
\end{tabular}




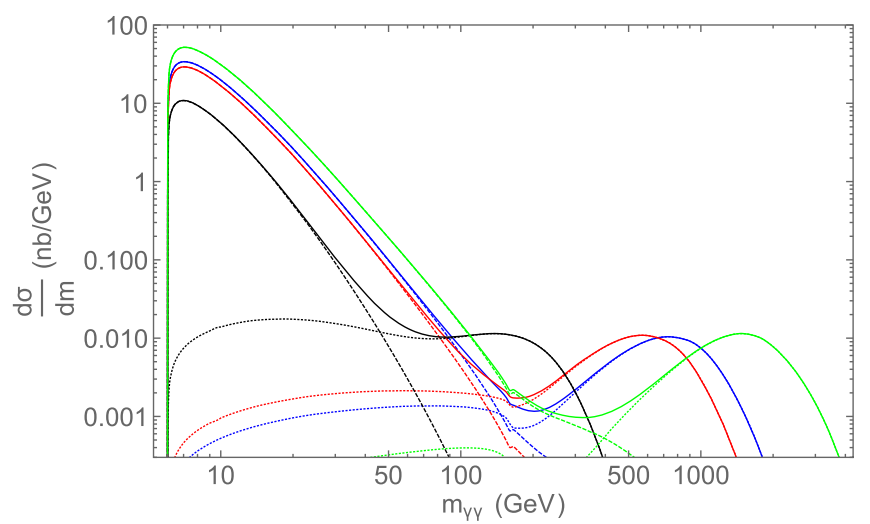

FIG. 3. Cross section versus diphoton invariant mass distribution in the future higher energy ATLAS $\mathrm{PbPb} \rightarrow \mathrm{PbPb} \gamma \gamma$ like experiments. Dashed curves are for SM contributions, dotted curves for NCQED, and solid correspond to the SM + NCQED, respectively. Black curves are for $\sqrt{\mathrm{s}_{\mathrm{NN}}}=5.02 \mathrm{TeV}, \Lambda_{\mathrm{NC}}=$ $100 \mathrm{GeV}$; red curves are for $\sqrt{\mathrm{S}_{\mathrm{NN}}}=25.10 \mathrm{TeV}, \Lambda_{\mathrm{NC}}=$ $257 \mathrm{GeV}$; blue curves are for $\sqrt{\mathrm{s}_{\mathrm{NN}}}=35.14 \mathrm{TeV}, \Lambda_{\mathrm{NC}}=$ $311 \mathrm{GeV}$, and green curves are for $\sqrt{\mathrm{s}_{\mathrm{NN}}}=100.40 \mathrm{TeV}$, $\Lambda_{\mathrm{NC}}=523 \mathrm{GeV}$.

NCQED contribution second peaks, as functions of $\left(\sqrt{\mathrm{s}_{\mathrm{NN}}}, \Lambda_{\mathrm{NC}}\right)$ follows more or less the similar sort of pattern. One can also notice in Fig. 3 that with increased collision energies the $\mathrm{NC}+\mathrm{NC}^{2}$ plateau and $\mathrm{NC}^{2}$ peak become more separated than in Fig. 2. Thus an small dent at $m_{\gamma \gamma} \in$ $[150,200] \mathrm{GeV}$ due to SM W-loops become visible in the total NCQED plots for next generation collider scenarios in Fig. 3, while only visible in the interference plots from Fig. 2. Such effect is unfortunately experimentally invisible. Similar high diphoton mass peak appeared in perturbatively expanded Born-Infeld theory [41], too. However, it is to be mentioned that in the latter case the peak actually locates in diphoton mass range close to/higher than the deformation parameter. Therefore, one has to extrapolate out of the perturbative regime (as the authors of [41] were aware themselves) to obtain credible results there. On the other hand, our results presented in this paper stay free from such a restriction as a consequence of the $\theta$-exact approach.
The integrated luminosity $\left(\gg 100 \mathrm{nb}^{-1}\right.$ ) required for detecting the NCQED second peak at the scale $\sim 0.01 \mathrm{nb} / \mathrm{GeV}$ is difficult to achieve for both the present day LHC $\left(>1 \mathrm{nb}^{-1} / \mathrm{yr}\right)$ and the future high luminosity (HL) LHC $\left(>3 \mathrm{nb}^{-1} / \mathrm{yr}\right)$ [57,58], respectively. However it would be attainable if the current performance projection (>30 nb $\mathrm{n}^{-1} / \mathrm{yr}$ ) of next generation hadron collider could be reached/exceeded [47]. It is also worthy to note that at each $\left.\mathrm{m}_{\gamma \gamma}\right|_{\max }$ 's from Tables I and II, in Figs. 1 and 3, the corresponding $\frac{\mathrm{d} \sigma_{S_{\mathrm{S}}}}{\mathrm{dm} \mathrm{m}_{v y}}$ values are several orders of magnitude below the second peaks.

In summary, our calculations show that ultraperipheral $\mathrm{PbPb} \rightarrow \mathrm{PbPb} \gamma \gamma$ scattering experiment on next generation hadron collider(s) could probe $\Lambda_{\mathrm{NC}}$ up to $\sim 300 \mathrm{GeV}$, if a high integrated luminosity $\gg 100 \mathrm{nb}^{-1}$ and proper diphoton invariant mass range $\left.\mathrm{m}_{\gamma \gamma}\right|_{\max } \gtrsim 3 \Lambda_{\mathrm{NC}}$ can be achieved by the technical design. Such performance would be close to the light-by-light scattering in the photon-photon mode of a linear $\mathrm{e}^{-} \mathrm{e}^{+}$collider. We therefore consider ultraperipheral heavy ion scattering a potential future alternative to photon-photon (mode of linear $\mathrm{e}^{-} \mathrm{e}^{+}$) collider in probing space-time noncommutativity on accelerators and wish this perspective can be included in the technical design considerations of the next generation hadron collider(s).

\section{ACKNOWLEDGMENTS}

J. T. would like to thank ATLAS collaboration colleagues, M. Dyndal and M. Schott, for discussions regarding $\mathrm{PbPb}$ collision experiments at the LHC. J. T. also acknowledge Wolfgang Hollik, Dieter Lust and Peter Minkowski for many discussions and to thank Max-Planck-Institute for Physics for hospitality. The work of R. H. has been supported by Croatian Science Foundation. The work of D. L. is supported by Serbian Ministry of Education, Science and Technological Development under the Project No. ON171031. Computation was done by the package LOOPTOOL [59] and Mathematica [60].
[1] H. S. Snyder, Quantized space-time, Phys. Rev. 71, 38 (1947).

[2] H. S. Snyder, The electromagnetic field in quantized spacetime, Phys. Rev. 72, 68 (1947).

[3] N. Seiberg and E. Witten, String theory and noncommutative geometry, J. High Energy Phys. 09 (1999) 032.

[4] J. Madore, S. Schraml, P. Schupp, and J. Wess, Gauge theory on noncommutative spaces, Eur. Phys. J. C 16, 161 (2000).
[5] R. Jackiw and S. Y. Pi, Covariant Coordinate Transformations on Noncommutative Space, Phys. Rev. Lett. 88, 111603 (2002).

[6] C.P. Martin and D. Sanchez-Ruiz, The One-Loop UV Divergent Structure of U(1) Yang-Mills Theory on Noncommutative $R^{4}$, Phys. Rev. Lett. 83, 476 (1999).

[7] R. J. Szabo, Quantum gravity, field theory and signatures of noncommutative spacetime, Gen. Relativ. Gravit. 42, 1 (2010). 
[8] X. Calmet, B. Jurco, P. Schupp, J. Wess, and M. Wohlgenannt, The standard model on non-commutative space-time, Eur. Phys. J. C 23, 363 (2002).

[9] W. Behr, N. Deshpande, G. Duplančić, P. Schupp, J. Trampetić, and J. Wess, The $Z \rightarrow \gamma \gamma, g g$ decays in the noncommutative standard model, Eur. Phys. J. C 29, 441 (2003).

[10] P. Aschieri, B. Jurco, P. Schupp, and J. Wess, Noncommutative GUTs, standard model and C, P, T, Nucl. Phys. B651, 45 (2003).

[11] I. Hinchliffe, N. Kersting, and Y. L. Ma, Review of the phenomenology of noncommutative geometry, Int. J. Mod. Phys. A 19, 179 (2004).

[12] P. Schupp and J. You, UV/IR mixing in noncommutative QED defined by Seiberg-Witten map, J. High Energy Phys. 08 (2008) 107.

[13] R. Horvat, D. Kekez, and J. Trampetic, Spacetime noncommutativity and ultra-high energy cosmic ray experiments, Phys. Rev. D 83, 065013 (2011).

[14] J. Trampetic and J. You, $\theta$-exact Seiberg-Witten maps at order $e^{3}$, Phys. Rev. D 91, 125027 (2015).

[15] J. Gomis and T. Mehen, Space-time noncommutative field theories and unitarity, Nucl. Phys. B591, 265 (2000).

[16] O. Aharony, J. Gomis, and T. Mehen, On theories with lightlike noncommutativity, J. High Energy Phys. 09 (2000) 023.

[17] S. M. Carroll, J. A. Harvey, V. A. Kostelecky, C. D. Lane, and T. Okamoto, Noncommutative Field Theory and Lorentz Violation, Phys. Rev. Lett. 87, 141601 (2001).

[18] M. Chaichian, M. M. Sheikh-Jabbari, and A. Tureanu, Hydrogen Atom Spectrum and the Lamb Shift in Noncommutative QED, Phys. Rev. Lett. 86, 2716 (2001).

[19] S. A. Abel, J. Jaeckel, V. V. Khoze, and A. Ringwald, Vacuum birefringence as a probe of Planck scale noncommutativity, J. High Energy Phys. 09 (2006) 074.

[20] R. Horvat and J. Trampetic, Constraining noncommutative field theories with holography, J. High Energy Phys. 01 (2011) 112.

[21] R. Horvat, A. Ilakovac, P. Schupp, J. Trampetić, and J. You, Yukawa couplings and seesaw neutrino masses in noncommutative gauge theory, Phys. Lett. B 715, 340 (2012).

[22] R. Horvat, J. Trampetic, and J. You, Spacetime deformation effect on the Early Universe and the PTOLEMY experiment, Phys. Lett. B 772, 130 (2017).

[23] R. Horvat, J. Trampetic, and J. You, Inferring type and scale of noncommutativity from the PTOLEMY experiment, Eur. Phys. J. C 78, 572 (2018).

[24] M. Hayakawa, Perturbative analysis on infrared aspects of noncommutative QED on $\mathrm{R} * * 4$, Phys. Lett. B 478, 394 (2000).

[25] M. Hayakawa, Perturbative analysis on infrared and ultraviolet aspects of noncommutative QED on $R^{4}$, arXiv:hep-th/ 9912167.

[26] C. P. Martin, J. Trampetic, and J. You, Super Yang-Mills and $\theta$-exact Seiberg-Witten map: Absence of quadratic noncommutative IR divergences, J. High Energy Phys. 05 (2016) 169.

[27] C.P. Martin, J. Trampetic, and J. You, Equivalence of quantum field theories related by the $\theta$-exact Seiberg-Witten map, Phys. Rev. D 94, 041703 (2016).
[28] C. P. Martin, J. Trampetic, and J. You, Quantum duality under the $\theta$-exact Seiberg-Witten map, J. High Energy Phys. 09 (2016) 052.

[29] C. P. Martin, J. Trampetic, and J. You, Quantum noncommutative ABJM theory: First steps, J. High Energy Phys. 04 (2018) 070.

[30] D. Lust and E. Palti, Scalar fields, hierarchical UV/IR mixing and the weak gravity conjecture, J. High Energy Phys. 02 (2018) 040.

[31] J. L. Hewett, F. J. Petriello, and T. G. Rizzo, Signals for noncommutative interactions at linear colliders, Phys. Rev. D 64, 075012 (2001).

[32] B. Badelek et al. (ECFA/DESY Photon Collider Working Group), TESLA: The superconducting electron positron linear collider with an integrated x-ray laser laboratory. Technical design report. Part 6. Appendices. Chapter 1. Photon collider at TESLA, Int. J. Mod. Phys. A 19, 5097 (2004).

[33] G. Jikia and A. Tkabladze, Photon-photon scattering at the photon linear collider, Phys. Lett. B 323, 453 (1994).

[34] G. J. Gounaris, P. I. Porfyriadis, and F. M. Renard, The $\gamma \gamma \rightarrow$ $\gamma \gamma$ process in the standard and SUSY models at highenergies, Eur. Phys. J. C 9, 673 (1999).

[35] Z. Bern, A. De Freitas, L. J. Dixon, A. Ghinculov, and H. L. Wong, QCD and QED corrections to light by light scattering, J. High Energy Phys. 11 (2001) 031.

[36] D. d'Enterria and G. G. daSilveira, Observing Light-byLight Scattering at the Large Hadron Collider, Phys. Rev. Lett. 111, 080405 (2013); 116, 129901(E) (2016).

[37] M. Klusek-Gawenda, P. Lebiedowicz, and A. Szczurek, Light-by-light scattering in ultraperipheral $\mathrm{Pb}-\mathrm{Pb}$ collisions at energies available at the CERN Large Hadron Collider, Phys. Rev. C 93, 044907 (2016).

[38] L. A. Harland-Lang, V. A. Khoze, and M. G. Ryskin, Exclusive LHC physics with heavy ions: SuperChic 3, Eur. Phys. J. C 79, 39 (2019).

[39] M. Aaboud et al. (ATLAS Collaboration), Evidence for light-by-light scattering in heavy-ion collisions with the ATLAS detector at the LHC, Nat. Phys. 13, 852 (2017).

[40] G. Aad et al. (ATLAS Collaboration), Observation of Lightby-Light Scattering in Ultraperipheral $\mathrm{Pb}+\mathrm{Pb}$ Collisions with the ATLAS Detector, Phys. Rev. Lett. 123, 052001 (2019).

[41] J. Ellis, N. E. Mavromatos, and T. You, Light-by-Light Scattering Constraint on Born-Infeld Theory, Phys. Rev. Lett. 118, 261802 (2017).

[42] P. Niau Akmansoy and L. G. Medeiros, Constraining nonlinear corrections to Maxwell electrodynamics using $\gamma \gamma$ scattering, Phys. Rev. D 99, 115005 (2019).

[43] V. A. Kostelecky and Z. Li, Gauge field theories with Lorentz-violating operators of arbitrary dimension, Phys. Rev. D 99, 056016 (2019).

[44] G. Baur, K. Hencken, D. Trautmann, S. Sadovsky, and Y. Kharlov, Coherent gamma gamma and gamma-A interactions in very peripheral collisions at relativistic ion colliders, Phys. Rep. 364, 359 (2002).

[45] N. Armesto, A. Dainese, D. d'Enterria, S. Masciocchi, C. Roland, C. Salgado, M. van Leeuwen, and U. Wiedemann, 
Heavy-ion physics studies for the Future Circular Collider, Nucl. Phys. A931, 1163 (2014).

[46] G.-Y. Qin, Physics with heavy-ion beams at SppC, Workshop on Physics at the CEPC/SppC (IHEP, Beijing, 2015).

[47] A. Dainese et al., Heavy Ions at the Future Circular Collider, CERN Yellow Reports (2017), p. 635-692, https://doi.org/10.23731/CYRM-2017-003.635.

[48] D. Latas, J. Trampetic, and J. You, Seiberg-Witten map Invariant Scatterings (to be published).

[49] S. Raju, The noncommutative S-matrix, J. High Energy Phys. 06 (2009) 005.

[50] J. H. Huang, R. Huang, and Y. Jia, Tree amplitudes of noncommutative U(N) Yang-Mills theory, J. Phys. A 44, 425401 (2011).

[51] A. C. Canbay, U. Kaya, B. Ketenoglu, B. B. Oner, and S. Sultansoy, SppC based energy frontier lepton-proton colliders: Luminosity and physics, Adv. High Energy Phys. 2017, 14021493 (2017).

[52] Y. C. Acar, A. N. Akay, S. Beser, A. C. Canbay, H. Karadeniz, U. Kaya, B. B. Oner, and S. Sultansoy, Future circular collider based lepton-hadron and photon-hadron colliders: Luminosity and physics, Nucl. Instrum. Methods Phys. Res., Sect. A 871, 47 (2017).
[53] A. Abada et al. (FCC Collaboration), FCC physics opportunities: Future collider conceptual design report Volume 1, Eur. Phys. J. C 79, 474 (2019).

[54] A. Abada et al. (FCC Collaboration), FCC-ee: The Lepton Collider: Future circular collider conceptual design report Volume 2, Eur. Phys. J. Special Topics 228, 261 (2019).

[55] A. Abada et al. (FCC Collaboration), FCC-hh: The Hadron Collider: Future circular collider conceptual design report Volume 3, Eur. Phys. J. Special Topics 228, 755 (2019).

[56] A. Abada et al. (FCC Collaboration), HE-LHC: The highenergy Large Hadron Collider: Future circular collider conceptual design report Volume 4, Eur. Phys. J. Special Topics 228, 1109 (2019).

[57] J. M. Jowett, M. Schaumann, and R. Versteegen, Heavy-ion operation of HL-LHC, Adv. Ser. Dir. High Energy Phys. 24, 359 (2015).

[58] J. Jowett et al., The 2018 heavy-ion run of the LHC, https:// doi.org/10.18429/JACoW-IPAC2019-WEYYPLM2

[59] T. Hahn and M. Perez-Victoria, Automatized one loop calculations in four-dimensions and D-dimensions, Comput. Phys. Commun. 118, 153 (1999).

[60] Wolfram Research, Inc., Mathematica, Version 12.0. Champaign, IL (2010). 